\title{
Processamento digital de imagens de plântulas na avaliação do vigor de sementes de Moringa oleifera Lam.
}

\author{
Digital processing of plant images in evaluating the vigor of Moringa oleifera \\ Lam. seeds
}

\section{Márcio Dias Pereira', João Antônio dos Reis" , Cibeli dos Santos Ferrari" ${ }^{I I}$, Alessandra Mendes Pacheco Guerra Vale ${ }^{\mathrm{IV}}$}

\begin{abstract}
Resumo
A utilização de técnicas que envolvam a análise computadorizada de plântulas na avaliação da qualidade de lotes de sementes tem se mostrado muito eficiente. Assim, objetivou-se, neste trabalho, verificar a eficiência do Sistema de Análise de Plântulas (SAPL) na detecção de diferenças de vigor entre lotes de sementes de moringa, em comparação às informações fornecidas por testes de vigor tradicionalmente utilizados. Para tanto, determinou-se o teor de água das sementes e a sua qualidade fisiológica pelos testes de germinação, primeira contagem de germinação, índice de velocidade de germinação, massa seca de plântulas, emergência, índice de velocidade de emergência, envelhecimento acelerado tradicional, envelhecimento acelerado com solução salina e utilizando-se o SAPL. A caracterização fisiológica das sementes determinou que os lotes 1 e 3 apresentaram qualidade superior aos demais, sendo o lote 4 o menos vigoroso. Os parâmetros obtidos na análise computadorizada (comprimento da parte aérea, comprimento da raiz primária, comprimento total de plântula, índice de crescimento, índice de uniformidade e índice de vigor) com o software SAPL foram eficientes para diferenciar os lotes de sementes em comparação aos testes tradicionalmente utilizados, não somente entre lotes de baixo e alto vigor, mas também para aqueles de qualidade intermediária, corroborando com os resultados obtidos nos testes tradicionalmente empregados na avaliação da qualidade de sementes.
\end{abstract}

Palavras-chave: Imagem de plântula; Germinação; Sistema automatizada

\begin{abstract}
The use of techniques involving a computerized analysis of seedlings in evaluating the seed lot quality has shown to be of great efficiency. Thus, the aim of this work was to verify the efficiency of the Seedling Analysis System (SAPL) in detecting vigor differences between moringa seed lots in comparison to the information provided by usual vigor tests. Therefore, the water content and the physiological quality of seeds by germination test, first count test, germination speed index, emergency speed index, traditional accelerated aging test, and accelerated aging test using saline solution were determined by SAPL. The experimental design was completely randomized, using four replicates. The parameters obtained in the computerized analysis (shoot and primary root lengths, total seedling length, and growth, uniformity, and vigor index) by the SAPL software were efficient in distinguishing seed lots in comparison to the usual tests, and not only among lots showing low and high vigor, but also for those showing intermediate quality. Keywords: Image analysis; Germination; Automated system

\footnotetext{
Engenheiro Agrônomo, Professor do Programa de Pós-graduação em Ciências Florestais, Escola Agrícola de Jundiaí, Universidade Federal do Rio Grande do Norte, Rodovia RN 160, Km 03, CEP 59280-000, Macaíba (RN), Brasil. marcioagron@gmail.com (ORCID: 0000-0001-9729-6503) Grande do Norte, Rodovia RN 160, Km 03, CEP 59280-000, Macaíba (RN), Brasil. joaoantonio.22@hotmail.com (ORCID: 0000-0003-4531-9720) Rio Grande do Norte, Rodovia RN 160, Km 03, CEP 59280-000, Macaíba (RN), Brasil. cibeliferrari@hotmail.com (ORCID: 0000-0003-2940-0190) 03, CEP 59280-000, Macaíba (RN), Brasil. alemendesp@gmail.com (ORCID: 0000-0003-3843-1327)
}

I Engenheiro Florestal, Estudante do Programa de Pós-graduação em Ciências Florestais, Escola Agrícola de Jundiaí, Universidade Federal do Rio

III Engenheiro Florestal, Pós-doutoranda do Programa de Pós-graduação em Ciências Florestais, Escola Agrícola de Jundiaí, Universidade Federal do

IV Engenheira de software, Professora Associada da Escola Agrícola de Jundiaí, Universidade Federal do Rio Grande do Norte, Rodovia RN 160, Km
\end{abstract}




\section{Introdução}

A Moringa oleifera Lam. (moringa) vem ganhando destaque, principalmente em regiões semiáridas, por se tratar de uma espécie que, além de se adaptar bem às condições de restrição hídrica, pode ser utilizada para fins farmacêuticos, alimentação humana e animal, como também em diferentes tipos de indústrias. Apesar da importância econômica da espécie em diferentes partes do mundo, ainda são escassos os estudos relacionados à tecnologia de produção e métodos padronizados para a avaliação da qualidade de suas sementes.

A qualidade da semente é compreendida por quatro atributos: genético, sanitário, físico e fisiológico (CARVALHO; NAKAGAWA, 2012). A avaliação do potencial fisiológico, que inclui o teste de germinação e a análise do vigor, é fundamental para a qualidade das sementes, pois fornece informações relacionadas à capacidade de desenvolvimento das plântulas, sob diferentes condições de ambiente, e à seleção de lotes de sementes para comercialização (MARCOS FILHO, 2015a). Entretanto, com o passar dos anos e o avanço das tecnologias, faz-se necessário o desenvolvimento e o aperfeiçoamento de novos métodos para determinar o vigor de lotes de sementes, sobretudo que confiram mais agilidade e rapidez ao processo de avaliação e análise.

Novas técnicas de avaliação da qualidade de sementes podem ser associadas a testes cujas metodologias já são consagradas, conferindo-lhes mais rapidez ou precisão. Entre os testes utilizados na análise de sementes, pode-se destacar o envelhecimento acelerado, utilizando água, ou o que substitui a água por solução saturada de $\mathrm{NaCl}$, um dos mais tradicionais e eficientes testes para a avaliação do vigor de sementes de várias espécies, em função da sua capacidade de proporcionar informações com alto grau de consistência (BAALBAKI et al., 2009). O envelhecimento acelerado é um teste fundamentado na aproximação de fatores ambientais adversos, como temperatura e umidade relativa elevada, que são tidos como responsáveis pela deterioração das sementes, sendo esse teste semelhante ao que acontece no envelhecimento natural das sementes, porém a uma velocidade acelerada, por meio da qual sementes menos vigorosas deterioram-se mais rapidamente do que as mais vigorosas, estabelecendo diferenças no potencial fisiológico das sementes analisadas (GUEDES et al., 2009a).

Embora os procedimentos para a realização dos vários testes de vigor estejam estabelecidos para grande parte das espécies conhecidas e forneçam dados confiáveis (MARCOS FILHO et al., 2009), uma das deficiências para a aplicação dos testes de vigor está relacionada com o tempo gasto para a execução e com a subjetividade da análise (MARCOS FILHO, 2015b). Contudo, técnicas de análises computadorizadas de imagens de plântulas associadas aos testes tradicionais podem minimizar essas limitações, pois se destacam por sua precisão e objetividade (SAKO et al., 2001; HOFFMASTER et al., 2003), entretanto, é necessário adaptar essas técnicas às diferentes espécies vegetais.

Dentre os softwares disponíveis no mercado, destaca-se o Seed Vigor Imaging System $\left(S_{V I S}{ }^{\circ}\right.$, criado pela Universidade de Ohio, que tem servido de base para a criação de outros sistemas semelhantes. Baseia-se na avaliação do vigor de sementes, a partir do desempenho de plântulas, mediante a coleta de dados referentes ao comprimento da raiz primária, do hipocótilo e da plântula total (raiz primária e hipocótilo), além da relação raiz/hipocótilo e de parâmetros baseados na velocidade e na uniformidade de crescimento (SAKO et al., 2001). Esse tipo de análise possibilita a determinação do vigor de várias sementes, como alface (SAKO et al., 2001), soja (HOFFMASTER et al., 2003), milho (GOMES JUNIOR et al., 2009) e mamona (KOBORI et al., 2010), mas são escassos estudos envolvendo sementes de espécies florestais.

A partir do software proposto por Sako et al. (2001), foi desenvolvido o Sistema de Análise de Plântulas (SAPL), que fornece dados do comprimento da parte aérea, raiz primária e total, índices de vigor, de crescimento e de uniformidade da plântula. Os valores do índice de vigor são fundamentados na rapidez e na uniformidade de desenvolvimento das plântulas da amostra, em relação ao máximo valor estimado para plântulas com a idade preestabelecida na programação do SAPL. A uniformidade é definida com base nos desvios do comprimento de cada plântula, 
em relação àquele máximo desenvolvido estimado. Dessa maneira, após o processamento das imagens, o software gera automaticamente valores numéricos referentes ao índice de crescimento, à uniformidade e ao vigor. Entre as diferenças do SVIS e o SAPL, pode-se destacar a forma de captação de imagens das plântulas, sendo, no primeiro, por meio de scanner e, no segundo, por fotografias digitais.

Sendo assim, objetivou-se, com o presente estudo, verificar a eficiência da utilização do Sistema de Análise de Plântulas (SAPL), para detectar diferenças de vigor entre lotes de sementes de moringa, a partir de plântulas obtidas nos testes de germinação e de envelhecimento acelerado.

\section{Material e métodos}

Utilizaram-se quatro lotes de sementes de moringa, cujos frutos foram colhidos manualmente, em árvores matrizes isoladas, no município de Upanema/RN. Cada lote reuniu sementes colhidas de seis árvores matrizes selecionadas ao acaso. A cada seis árvores, formavase um lote, até que os quatro lotes de sementes estivessem colhidos.

Após a colheita, os frutos foram levados ao laboratório, e as suas sementes removidas. Procedeu-se a retirada manual das alas e posterior armazenagem em sacos de papel kraft (46 x 21 $\mathrm{x} 13 \mathrm{~cm}$ ), os quais foram mantidos em condições normais de laboratório ( $\mathrm{T}=28 \pm 2{ }^{\circ} \mathrm{C}$; U.R. $=54$ $\pm 16 \%)$ até o momento das avaliações.

A determinação do teor de água das sementes foi realizada pelo método da estufa a $105 \pm 3$ ${ }^{\circ} \mathrm{C}$, utilizando-se, para cada lote, duas repetições de 15 sementes acondicionadas em recipientes metálicos, durante 24 horas. Também foram obtidos os teores de água das sementes antes e após exposição ao envelhecimento acelerado (tradicional e com solução salina saturada). Os resultados foram expressos pela média aritmética das repetições em porcentagens das amostras (base úmida) (BRASIL, 2009).

A qualidade fisiológica das sementes de cada lote foi avaliada por meio dos testes explicitados a seguir.

Teste de germinação e primeira contagem de germinação: quatro repetições de 25 sementes para cada lote foram distribuídas em rolos de papel toalha $\left(\right.$ Germitest $\left.^{\circledR}\right)$, umedecidos com água destilada, na quantidade equivalente a 2,5 vezes o peso do papel seco, acrescida de $2 \mathrm{~mL}$ de nistatina (fungicida) por litro de solução. Os rolos foram acomodados em sacos plásticos transparentes (24 x $34 \mathrm{~cm}$ ) e acondicionados em germinador tipo B.O.D. (BiochemicalOxygenDemand), regulado à temperatura de $30{ }^{\circ} \mathrm{C}$ por 12 dias. As contagens das sementes germinadas foram realizadas no $7^{\circ}$ (primeira contagem) e no $12^{\circ}$ dia (germinação) após a instalação do teste. Os resultados foram expressos em porcentagem de plântulas normais, de acordo com as recomendações para a espécie, sugeridas por Bezerra et al. (2004).

Velocidade de germinação: obtida a partir da fórmula proposta por Maguire (1962) para o cálculo do índice de velocidade de germinação (IVG), e conduzido junto com o teste de germinação, com contagem diária do número de sementes que emitiram raiz primária igual ou superior a $1 \mathrm{~mm}$.

Massa seca da plântula (MS): quatro repetições de dez plântulas de cada lote foram colocadas em sacos de papel $(40 \times 60 \mathrm{~cm})$ e levadas à estufa regulada a $105^{\circ} \mathrm{C}$ por 24 horas. Em seguida, foram colocadas para resfriar e pesadas em balançade precisão $0,001 \mathrm{~g}$, determinandose o peso da massa seca total(mg plântula ${ }^{-1}$ ) (NAKAGAWA, 1999b).

Emergência de plântulas: foi conduzido em casa de vegetação, com quatro repetições de 25 sementes para cada lote, distribuídos em bandejas plásticas $(15 \times 21 \times 5 \mathrm{~cm})$ contendo areia fina $(0,2 \mathrm{~mm})$. A porcentagem de emergência de plântulas foi determinada após 14 dias.

Velocidade de emergência: realizada concomitantemente ao teste de emergência e determinada a partir da contagem diária do número de plântulas emergidas, sendo o índice de velocidade de emergência (IVE) calculadode acordo com a fórmula proposta por Maguire (1962).

Envelhecimento acelerado tradicional (EAT):uma camada de sementes foi distribuída 
sobre uma tela de metal acoplada a uma caixa plástica do tipo gerbox (11 x $11 \times 3,5 \mathrm{~cm})$ contendo $40 \mathrm{~mL}$ de água destilada. Cada caixa foi coberta e exposta à temperatura de $40{ }^{\circ} \mathrm{C}$ por 72 horas, em germinadores do tipo B.O.D. (MEDEIROS, 2015). Posteriormente, determinou-se o teor de água e a porcentagem de germinação com contagem final após oito dias.

Envelhecimento acelerado com água salina (EAAS): conduzido de forma semelhante ao procedimento tradicional, porém com o uso de $40 \mathrm{~mL}$ de solução saturada de $\mathrm{NaCl}(40 \mathrm{~g}$ de $\mathrm{NaCl}$ em 100 mL de água) em substituição à água destilada (JIANHUA; MCDONALD, 1996).

Análise computadorizada de imagens de plântulas pelo SAPL:trata-se de um sistema automatizado de análise de plântulas, a partir de imagens digitais, desenvolvido pelo Grupo de Estudos em Tecnologia de Sementes (GETSem) na Escola Agrícola de Jundiaí/Universidade Federal do Rio Grande do Norte (EAJ/UFRN). Para as análises, quatro repetições de 20 plântulas de cada lote de sementes, provenientes dos testes de germinação, envelhecimento acelerado tradicional e envelhecimento acelerado com solução salina, após oito, dez e doze dias da instalação dos testes de germinação, foram submetidas à análise de vigor utilizando-se o SAPL.

Para que as imagens pudessem ser capturadas por meio de fotografia digital, as plântulas de cada repetição foram transferidas do papel toalha $\left(\right.$ Germitest $\left.^{\circ}\right)$ para uma folha de papel EVA de coloração azul $(40$ x $60 \mathrm{~cm})$, contendo dez células $(5 \mathrm{~cm}$ de largura cada), separadas por uma faixa branca. Cada plântula foi colocada em uma célula (na primeira célula, da direita para a esquerda, colocou-se uma métrica formada por dois pontos distantes um centímetro do outro, além da identificação das plântulas e das repetições) (Figura 1).

\section{Figura 1 - Fundo azul de papel EVA para captação de imagens com as plântulas a serem analisadas pelo SAPL}

Figure 1 - Blue-background EVA paper to capture seedling images to be analyzed by SAPL

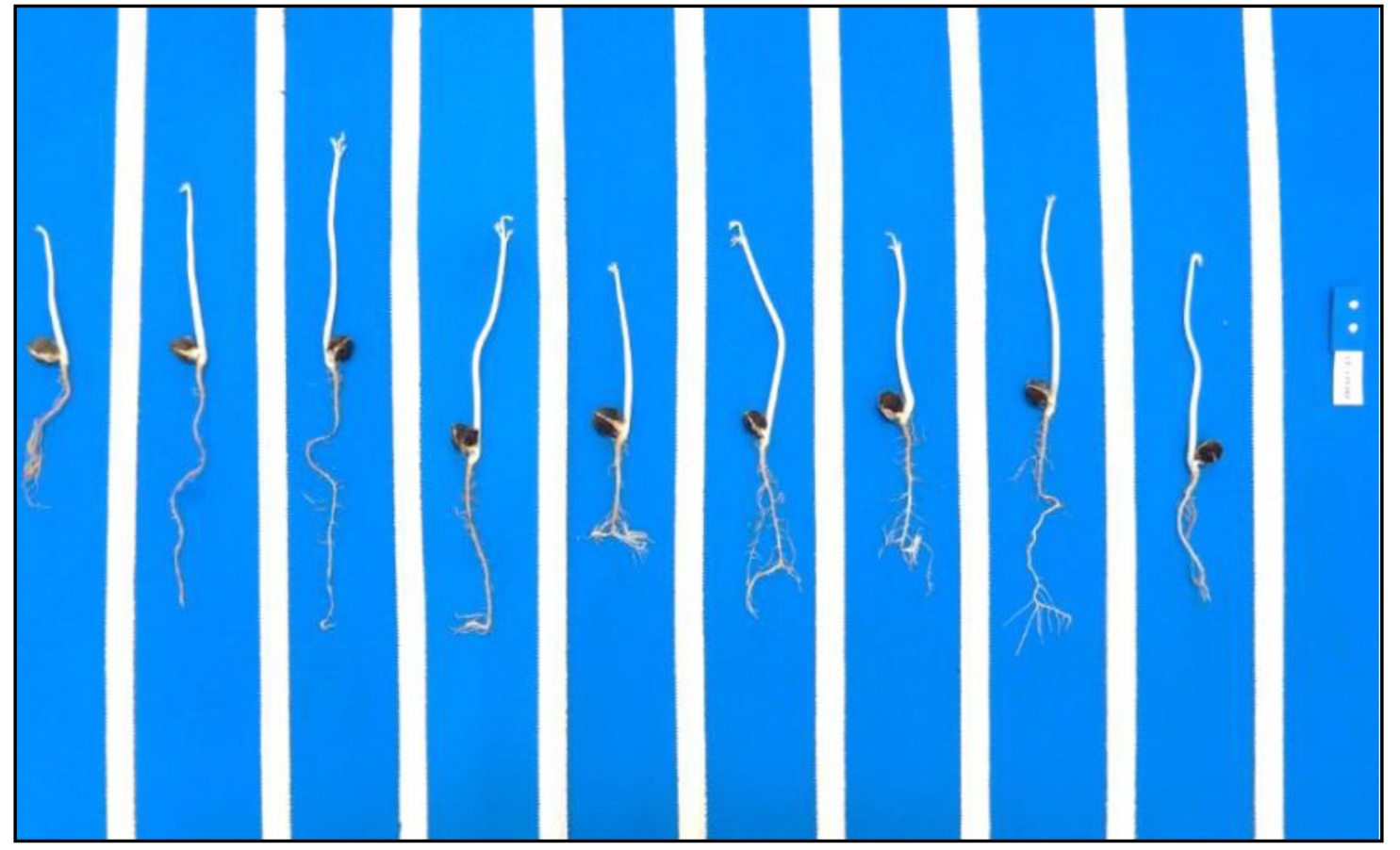

Fonte: Autores (2020)

As imagens foram captadas com uma câmera digital do tipo semiprofissional, modelo Sony Nex-F3 (sensor APS-C HD de 16.1 MP, com lentes 18-55 mm f/3.5-5.6). Após a captação, as imagensforam transferidas para um computador, utilizando-se o software Play Memories Home 
Versão 1.2.00.03162 e salvas com a resolução 16.1 MP, no formato JPEG. As imagens foram salvas em pastas identificadas para cada repetição. Posteriormente, elas foram analisadas utilizando-se o software SAPL, instalado em um computador de modelo HP Compaq Pro 630S SFF, processador AMD A6- 5400B APU WITH Radeon (tm) HD Graphics de 3,60 GHz, 4 GB RAM e HD de 500Gb, Sistema Operacional de 64 Bits, operado pelo sistema Windows 7 Professional.

Ao iniciar o programa, após a tela inicial, selecionou-se a espécie que seria analisada. Em seguida, preencheram-se os valores mínimos, determinados para a espécie, do comprimento da parte aérea e da raiz primária para uma plântula da espécie. Para a moringa, considerouse como tamanho mínimo de plântula aceitável 0,4 e $0,2 \mathrm{~cm}$ para a parte aérea e para a raiz, respectivamente, de acordo com Rocha (2015). Na mesma tela, preencheu-se o peso do crescimento e da uniformidade no cálculo do índice de vigor, que, neste trabalho, foram definidos na proporção 70:30 (crescimento: uniformidade), como proposto por Gomes Junior et al. (2009). Depois de registrados esses valores, iniciou-se o carregamento das imagens das plântulas por repetição e, posteriormente, o processamento de cada plântula a ser analisada. Os resultados foram automaticamente salvos em um arquivo do tipo Excel e arquivados na pasta correspondente ao lote analisado.

Os arquivos forneceram os resultados da avaliação das plântulas, sendo comprimento da parte aérea, da raiz primária e da plântula inteira, além dos índices de crescimento (IC), uniformidade (IU) e de vigor (IV), de acordo com os cálculos propostos por Sako et al. (2001) e gerados automaticamente pelo programa.

O delineamento experimental utilizado foi o inteiramente casualizado com quatro repetições por tratamento. Os dados foram submetidos à análise de variância (ANOVA) e, ao se verificar efeito significativo dos tratamentos, as médias foram comparadas pelo teste de Tukey, em nível de 1 e $5 \%$ de probabilidade, sendo os dados de porcentagem transformados em arc seno $\sqrt{ }(\mathrm{x} / 100)$, com os resultados expressos pelos números originais, sem a transformação. Utilizou-se o programa estatístico SAS (SAS INSTITUTE, 2000).

\section{Resultados e discussão}

A germinação e o IVG foram os testes que mais se assemelharam no ranqueamento dos lotes, sendo que os lotes 1 e 3 apresentaram valores superiores aos demais (Tabela 1). O lote 4 apresentou o pior desempenho, entretanto observou-se diferença na classificação da primeira e da segunda ordem entre os testes de germinação e IVG, com o teste de emergência. Na PCG e MS, o lote 1 apresentou os maiores valores. No EAAS, o lote 2 apresentou maior vigor, e os lotes 3 e 4 foram os que apresentaram pior desempenho.

$\mathrm{O}$ vigor das sementes está associado diretamente à influência do ambiente e, por conseguinte, testes baseados no desenvolvimento de plântulas em laboratório, como primeira contagem, massa seca e IVG podem não apresentar sensibilidade para detectar diferenças pouco acentuadas no potencial fisiológico das sementes (MARCOS FILHO, 2015b).

Estudos realizados por Guedes et al. (2009b) confirmaram que os testes de emergência e o índice de velocidade de emergência foram eficientes para a determinação do vigor de lotes de sementes de Erytrhina velutina Willd (mulungu), sugerindo que esse teste é eficiente para a distinção do vigor entre lotes de sementes, como ocorreu no presente trabalho.

O teste de emergência em campo é um indicativo da eficiência dos testes para avaliação do potencial fisiológico de lotes de sementes, pois, na sua realização, devem ser fornecidas às sementes condições que simulem aquelas que elas encontrarão por ocasião da semeadura em campo (MARCOS FILHO, 2015a).

De acordo com Baalbaki et al. (2009), o teste de envelhecimento acelerado com solução saturada de $\mathrm{NaCl}$ é um dos mais eficientes para determinar o vigor entre os lotes. Neste trabalho, as sementes de Moringa oleifera apresentaram redução na qualidade fisiológica quando foram submetidas ao envelhecimento acelerado tradicional e com solução salina de $\mathrm{NaCl}$, provavelmente 
devido aos danos causados na permeabilidade da membrana celular (Tabela 1). Vieira e Carvalho (1994) afirmam que as alterações no metabolismo, nos solutos e na troca de água entre as células e o ambiente externo são modificações degenerativas que indicam a perda de viabilidade da semente. Resultados semelhantes foram obtidos com sementes da Chorisia speciosa St. Hil. (paineira) (FANTI; PEREZ, 2005), Caesalpinia peltophoroides Benth. (coração-negro) (PONTES et al., 2006) e Schinus terebinthifolius Raddi (aroeira-vermelha) (PACHECO et al., 2011).

Tabela 1 - Germinação (G), primeira contagem (PCG), índice de velocidade de germinação (IVG), massa seca de plântulas em laboratório (MS), emergência (E), índice de velocidade de emergência (IVE), germinação após o envelhecimento acelerado tradicional (EAT) e germinação após o envelhecimento acelerado com água salina (EAAS) de quatro lotes de sementes de Moringa oleifera

Table 1 - Germination (G), first count germination (FCG), germination speed index (GSI), dry weight of seedlings in laboratory (DW), emergence (E), emergence speed index (ESI), germination after traditional accelerated aging (TAA), and germination after accelerated aging using saline water (AASW) from four lots of Moringa oleifera seeds

\begin{tabular}{lcccccccc}
\hline Lotes & G (\%) & PCG (\%) & IVG & MS $\left(\mathbf{m g ~ p l}^{-1}\right)$ & E (\%) & IVE & EAT (\%) & EAAS (\%) \\
\hline $\mathbf{1}$ & $76,66 \mathrm{a}$ & $12,50 \mathrm{a}$ & $4,80 \mathrm{a}$ & $165,09 \mathrm{a}$ & $63,33 \mathrm{~b}$ & $2,47 \mathrm{ab}$ & $52,50 \mathrm{ab}$ & $41,67 \mathrm{~b}$ \\
$\mathbf{2}$ & $32,50 \mathrm{c}$ & $3,33 \mathrm{c}$ & $2,30 \mathrm{c}$ & $154,78 \mathrm{~b}$ & $55,42 \mathrm{c}$ & $2,74 \mathrm{a}$ & $26,67 \mathrm{a}$ & $50,00 \mathrm{a}$ \\
$\mathbf{3}$ & $56,67 \mathrm{~b}$ & $6,67 \mathrm{~b}$ & $3,94 \mathrm{~b}$ & $138,15 \mathrm{c}$ & $66,69 \mathrm{a}$ & $2,68 \mathrm{a}$ & $45,00 \mathrm{a}$ & $28,34 \mathrm{c}$ \\
$\mathbf{4}$ & $16,67 \mathrm{~d}$ & $3,33 \mathrm{c}$ & $1,18 \mathrm{~d}$ & $155,84 \mathrm{~b}$ & $46,69 \mathrm{~d}$ & $1,99 \mathrm{~b}$ & $20,00 \mathrm{~b}$ & $29,17 \mathrm{c}$ \\
$\mathbf{F}$ & $344,85^{* *}$ & $726^{* *}$ & $172,84^{* *}$ & $38,27^{* *}$ & $1824,24^{* *}$ & $6,15^{* *}$ & $6,15^{*}$ & $53,80^{* *}$ \\
$\mathbf{d m s}$ & 5,97 & 2,07 & 0,51 & 7,61 & 0,87 & 0,57 & 0,57 & 5,97 \\
$\mathbf{C V}(\%)$ & 6,24 & 5,42 & 8,10 & 2,36 & 0,72 & 11,06 & 11,05 & 7,63 \\
\hline
\end{tabular}

Fonte: Autores (2020)

Em que: médias seguidas pela mesma letra não diferem estatisticamente entre si pelo teste de Tukey $(P<0,05)$;

** significativo em nível de $1 \%$ de probabilidade $(\mathrm{p}<0,01)$; * significativo em nível de $5 \%$ de probabilidade $(.01$ $=<\mathrm{p}<0,05) ;{ }^{\mathrm{ns}}$ não significativo ( $\left.\mathrm{p}>=0,05\right)$.

Na Tabela 2, são apresentados os teores de água das sementes antes e após a exposição ao envelhecimento acelerado tradicional (EAT) e ao envelhecimento acelerado com água salina (EAAS). A uniformização do teor de água das sementes é essencial para a padronização das avaliações e para a obtenção de resultados consistentes (SILVA; CICERO, 2014). Os valores dos teores de água não foram avaliados estatisticamente, sendo utilizados apenas para a caracterização inicial e o controle dos lotes após a realização dos testes de envelhecimento acelerado tradicional e com $\mathrm{NaCl}$. Observou-se aumento do teor de água das sementes, em função do envelhecimento acelerado, seja pelo método tradicional, seja com água salina. O aumento excessivo de água nas sementes pode provocar danos, ocasionando, por exemplo, um processo de deterioração mais precoce, ou mesmo o favorecimento e o desenvolvimento de patógenos, acarretando perda do poder germinativo e do vigor das sementes (CARVALHO; NAKAGAWA, 2012). 


\section{Tabela 2 - Teor de água das sementes antes (TA) e após o envelhecimento acelerado tradicional (EAT) e o envelhecimento acelerado com água salina (EAAS) de quatro lotes de sementes de Moringa oleifera}

Table 2 - Seed water content before (WC) and after traditional accelerated aging (TAA), and seed water content before and after accelerated aging using saline water (AASW) from four lots of Moringa oleifera seeds

\begin{tabular}{lccc}
\hline Lotes & TA (\%) & EAT (\%) & EAAS (\%) \\
\hline $\mathbf{1}$ & 7,28 & 22,01 & 8,12 \\
$\mathbf{2}$ & 7,48 & 30,58 & 5,15 \\
$\mathbf{3}$ & 7,60 & 24,97 & 12,76 \\
$\mathbf{4}$ & 7,40 & 29,55 & 8,24 \\
\hline
\end{tabular}

Fonte: Autores (2020)

Dentre as vantagens da utilização de soluções de $\mathrm{NaCl}$, destaca-se a uniformização do teor de água das sementes, de modo que os teores de água permanecem em níveis inferiores, suficientes para impedir o desenvolvimento de microrganismos (JIANHUA; MCDONALD, 1996; MARCOS FILHO, 2015a). Os resultados da avaliação das plântulas, obtidas após o $8^{\circ}, 10^{\circ}, 12^{\circ}$ dia da instalação do teste de germinação, do teste de envelhecimento acelerado tradicional e do teste de envelhecimento com solução salina, analisadas pelo SAPL, estão apresentados nas Tabelas 3, 4 e 5, respectivamente.

Para as plântulas obtidas no $10^{\circ}$ dia após a instalação do teste de germinação (Tabela 3), o comprimento da parte aérea (CPA), no lote 1, apresentou o maior comprimento, e o lote 2, o menor. Para o comprimento da raiz primária (CRP) e da plântula total (CPT), verificou-se que o ranqueamento dos lotes ocorreu de forma semelhante, observando-se superioridade para o lote 1 em relação aos demais lotes. Para o índice de uniformidade (IU) e de vigor (IV), os lotes 1 e 3 apresentaram os valores mais altos, enquanto o índice de crescimento (IC) detectou superioridade do lote $1 \mathrm{e}$ inferioridade do lote 2 . Nota-se que o lote 1 foi superior na maioria dos parâmetros obtidos no SAPL, a partir de plântulas oriundas do teste de germinação após oito dias da sua instalação.

As taxas de comprimento de plântulas de Eucalyptus via scanner de tela plana, auxiliada por software analítico ScionImage, também permitiram medições precisas do comprimento de raízes (DORNELAS; LOBO; VIEIRA, 2005).

Segundo Nakagawa (1999a), o teste de comprimento de plântulas tem como objetivo caracterizar o vigor de lotes de sementes pela avaliação do comprimento médio das plântulas normais ou de partes destas, a exemplo da raiz primária e epicótilo, a partir de sementes colocadas para germinar em condições controladas de laboratório, em geral, as mesmas empregadas para o teste de germinação. Guedes et al. (2013), em estudos com sementes de Amburana cearensis (cumaru), observaram que o comprimento das plântulas também pode ser eficiente quando aplicado para avaliação de plântulas oriundas do teste de emergência em campo.

A utilização do índice de crescimento também foi eficaz para a avaliação do vigor de sementes de Impatiens wallerana (beijo-turco) (OAKLEY et al., 2003), em um sistema de análise computadorizada de imagens de plântulas semelhante ao SAPL. Segundo esses autores, a avaliação do crescimento de plântulas após a germinação pode ser considerado um teste de vigor significativo. Há um fundamento de que o crescimento das plântulas diminui com o aumento da deterioração das sementes, e que os lotes de sementes mais vigorosas devem deteriorar-se de modo mais lento que as menos vigorosas (SILVA; CICERO, 2014).

No teste com sementes de moringa submetidas ao envelhecimento acelerado tradicional, analisadas pelo SAPL, após oito dias da instalação do teste (Tabela 3), observa-se que, para todos os comprimentos (CPA, CRP e CPT) e para o IC, há inferioridade do lote 3 em relação aos demais lotes, 
entretanto observa-se uma concordância no ranqueamento do CRP, CPT e o IC. O IU detectou que o lote 4 apresentou um desempenho inferior em relação aos demais lotes. O IV foi menos sensível para detectar diferença de vigor entre os lotes, pois só conseguiu ranquear os melhores lotes (lote 1 e 3 ) e os piores lotes (lote 2 e 4).

Para as sementes submetidas ao envelhecimento acelerado com solução de $\mathrm{NaCl}$ e analisadas pelo sistema SAPL, com oito dias de idade (Tabela 3), observa-se que o CPA do lote 2 apresentou-se superior aos demais lotes, entretanto, no lote 3 , observou-se maiores comprimentos no CRP e no CPT e maior índice de crescimento (IC) em relação aos demais lotes. O lote 2 apresentou inferioridade quando analisado pelo IU e IV. Percebe-se que o lote 3 foi superior na maioria dos parâmetros do teste de envelhecimento acelerado com solução salina de $\mathrm{NaCl}$, após oito dias.

Tabela 3 - Comprimento da parte aérea (CPA), da raiz primária (CRP) da plântula total (CPT), índices de uniformidade (IU), crescimento (IC) e vigor (IV) de quatro lotes de sementes de Moringa oleifera utilizando o software SAPL, a partir de plântulas obtidas pelo teste de germinação, pelo teste de germinação de sementes submetidas ao teste de envelhecimento acelerado tradicional e pelo teste de germinação de sementes submetidas ao teste de envelhecimento acelerado com solução salina de $\mathrm{NaCl}$, na primeira época de avaliação

Table 3 - Shoot (SL), first root (FRL), total seedling (TSL) length; Uniformity (UI), growth (GI), and vigor (VI) indices from four lots of Moringa oleifera seeds using the SAPL software from seedlings obtained by the germination test, germination test from seeds submitted to traditional accelerated aging, and from seeds submitted to accelerated aging using $\mathrm{NaCl}$ saline water at the first evaluation time

\begin{tabular}{|c|c|c|c|c|c|c|}
\hline \multicolumn{7}{|c|}{ Teste de germinação } \\
\hline \multirow{2}{*}{ Lote } & CPA & CRP & CPT & \multirow{2}{*}{ IU } & \multirow{2}{*}{ IC } & \multirow{2}{*}{ IV } \\
\hline & \multicolumn{3}{|c|}{$\mathrm{Cm}$} & & & \\
\hline 1 & 4,27 a & $7,21 \mathrm{a}$ & 11,49 a & $850,89 \mathrm{~b}$ & $461,30 \mathrm{a}$ & $578,18 \mathrm{a}$ \\
\hline 2 & $3,30 \mathrm{c}$ & $4,81 \mathrm{~b}$ & $8,11 \mathrm{~b}$ & $896,33 \mathrm{ab}$ & $325,20 \mathrm{c}$ & $496,54 \mathrm{~b}$ \\
\hline 3 & $3,34 \mathrm{bc}$ & $5,34 \mathrm{~b}$ & $8,32 \mathrm{~b}$ & 911,31 a & $408,00 \mathrm{ab}$ & $558,99 \mathrm{a}$ \\
\hline 4 & $4,11 \mathrm{ab}$ & $4,52 \mathrm{~b}$ & $8,64 \mathrm{~b}$ & $773,42 \mathrm{c}$ & $361,28 \mathrm{bc}$ & $484,92 \mathrm{~b}$ \\
\hline $\mathbf{F}$ & $7,20^{* *}$ & $20,96^{* *}$ & $15,06^{* *}$ & $31,95^{* *}$ & $18,38^{* *}$ & $40,12^{* *}$ \\
\hline dms & 0,79 & 1,11 & 1,71 & 46,02 & 57,75 & 30,41 \\
\hline CV \% & 10,1 & 9,65 & 8,91 & 2,55 & 7,07 & 2,73 \\
\hline \multicolumn{7}{|c|}{ Teste de envelhecimento acelerado tradicional } \\
\hline \multirow{2}{*}{ Lote } & CPA & CRP & CPT & \multirow{2}{*}{ IU } & \multirow{2}{*}{ IC } & \multirow{2}{*}{ IV } \\
\hline & & $\mathrm{Cm}$ & & & & \\
\hline 1 & $2,45 \mathrm{~b}$ & 5,99 a & $8,45 \mathrm{a}$ & $965,35 \mathrm{a}$ & $360,97 \mathrm{a}$ & $578,18 \mathrm{a}$ \\
\hline 2 & $3,09 \mathrm{a}$ & $5,51 \mathrm{a}$ & $8,62 \mathrm{a}$ & $905,08 \mathrm{~b}$ & $353,40 \mathrm{a}$ & $496,54 \mathrm{~b}$ \\
\hline 3 & $1,60 \mathrm{c}$ & $2,21 \mathrm{c}$ & $3,50 \mathrm{c}$ & $969,25 \mathrm{a}$ & $172,30 \mathrm{c}$ & $558,99 \mathrm{a}$ \\
\hline 4 & $2,57 \mathrm{~b}$ & $3,82 \mathrm{~b}$ & $6,39 \mathrm{~b}$ & 895,39 c & $284,10 \mathrm{~b}$ & $484,92 \mathrm{~b}$ \\
\hline $\mathbf{F}$ & $57,28^{\star \star *}$ & $195,73^{* *}$ & $840,36^{* *}$ & $683,41^{* *}$ & $419,14^{* *}$ & $40,12^{* *}$ \\
\hline $\mathrm{dms}$ & 0,33 & 0,51 & 0,34 & 6,25 & 17,92 & 30,41 \\
\hline CV \% & 6,76 & 5,63 & 2,44 & 0,32 & 2,92 & 2,73 \\
\hline
\end{tabular}


Tabela 3 - Conclusão ...

Table 3 - Conclusion ...

\begin{tabular}{|c|c|c|c|c|c|c|}
\hline \multicolumn{7}{|c|}{ Teste de envelhecimento acelerado com solução salina } \\
\hline \multirow{2}{*}{ Lote } & CPA & CRP & CPT & \multirow{2}{*}{ IU } & \multirow{2}{*}{ IC } & \multirow{2}{*}{ IV } \\
\hline & & $\mathbf{C m}$ & & & & \\
\hline 1 & $4,12 \mathrm{ab}$ & $7,75 \mathrm{ab}$ & $11,84 \mathrm{ab}$ & 848,86 a & $489,10 \mathrm{~b}$ & $597,03 \mathrm{~b}$ \\
\hline 2 & $4,94 \mathrm{a}$ & $6,90 \mathrm{~b}$ & $11,85 \mathrm{ab}$ & $792,06 \mathrm{~b}$ & $457,80 \mathrm{~b}$ & $558,08 \mathrm{bc}$ \\
\hline 3 & $4,06 \mathrm{~b}$ & $9,39 \mathrm{a}$ & 13,46 a & $850,91 \mathrm{a}$ & 606,60 a & 679,86 a \\
\hline 4 & $3,84 \mathrm{~b}$ & $6,49 \mathrm{~b}$ & $10,34 \mathrm{~b}$ & $860,00 \mathrm{a}$ & $464,20 \mathrm{~b}$ & $582,92 \mathrm{~b}$ \\
\hline $\mathbf{F}$ & $5,96^{* *}$ & $6,83^{* *}$ & $3,63^{*}$ & $17,35^{* *}$ & $7,14^{4 * *}$ & $1266,73^{4 * *}$ \\
\hline dms & 0,83 & 2,06 & 2,80 & 21,23 & 10,91 & 36,40 \\
\hline CV \% & 9,34 & 12,86 & 11,25 & 1,78 & 10,30 & 1,96 \\
\hline
\end{tabular}

Fonte: Autores (2020)

Em que: médias seguidas pela mesma letra não diferem estatisticamente entre si pelo teste de Tukey $(P<0,05)$;

** significativo em nível de $1 \%$ de probabilidade $(\mathrm{p}<.01)$; * significativo em nível de $5 \%$ de probabilidade $(.01=<$ $\mathrm{p}<.05) ;{ }^{\text {ns }}$ não significativo ( $\left.\mathrm{p}>=.05\right)$.

$\mathrm{Na}$ Tabela 4, observam-se os resultados da avaliação do potencial fisiológico das sementes de moringa, em plântulas após dez dias da instalação do teste de germinação e analisadas por meio do softwareSAPL. Dentre as avaliações, nota-se que o CRP foi a variável mais sensível, pois conseguiu diferenciar todos os lotes. O lote 3, em todos os comprimentos analisados (CPA, CRP e CPT), apresentou crescimentos superiores quando comparado com os outros lotes. Quando avaliado o IU, notou-se que o lote 2 apresentou maior índice, e o lote 4, menor. O IC e o IV não conseguiram apresentar diferenças entre os lotes.

Em relação à moringa, o desenvolvimento de plântula com sistema radicular mais acentuado, oriundos de sementes mais vigorosas, pode ser uma característica favorável à adaptação e à sobrevivência da espécie, quando esta iniciar o processo de crescimento, visto que haverá maior velocidade nos processos metabólicos, propiciando emissão mais rápida e uniforme da raiz primária, de modo que um sistema radicular mais vigoroso será eficaz para absorção de água e nutrientes, e com maiores taxas de crescimento e produção (ROCHA et al., 2016).

Quando se avaliou o vigor de sementes de moringa submetidas ao envelhecimento acelerado tradicional, analisadas pelo SAPL, após dez dias da instalação do teste de germinação posterior ao envelhecimento (Tabela 4), observou-se que o CPA e o CPT do lote 3 diferenciaramse dos demais lotes, sendo o lote com maior comprimento da parte aérea e total. O CRP expressou pouca sensibilidade na diferenciação dos lotes, destacando-se apenas a inferioridade do lote 4, em relação aos demais lotes. O lote 3 expressou menor índice de uniformidade (IU). Para os IC e IV, não foram detectadas diferenças estatísticas entre os lotes testados.

Sementes de moringa submetidas ao envelhecimento acelerado com solução salina de $\mathrm{NaCl}$, analisadas pelo sistema SAPL, após dez dias de idade (Tabela 4), acusaram inferioridade do lote 2 para o CPA e o CPT, quando comparado aos demais. O CRP e o IV não apresentaram diferenças significativas entre os lotes. O lote 2 também se diferenciou para os índices de uniformidade (IU) e crescimento (IC), pois o IU apresentou valor superior aos demais lotes, enquanto no IC observou-se valores menores.

A análise computadorizada pelo SAPL, nas condições testadas e cujos resultados se encontram na Tabela 4, não detectou diferenças significativas nos índices de vigor (IV), tanto para germinação como para envelhecimento acelerado tradicional e com solução salina. Estudos 
realizados por Marcos Filho et al. (2009) sobre os métodos para avaliação de vigor, incluindo análise computadorizada de imagens, atestaram que o índice de vigor pode ser ineficiente ao determinar o vigor de sementes de soja, pois um conjunto de condições devem favorecer a expressão do vigor e sua captação pelos sistemas automatizados. Apesar disso, segundo Bennett (2002), quando bem conduzida, a determinação desse índice expressa maior sensibilidade para caracterizar o aspecto fisiológico da qualidade de sementes do que o teste de germinação, fornecendo resposta consistente com o ranqueamento dos lotes para muitas espécies.

Tabela 4 - Comprimento da parte aérea (CPA), da raiz primária $(\mathrm{CRP})$ da plântula total (CPT), índices de uniformidade (IU), crescimento (IC) e vigor (IV) de quatro lotes de sementes de Moringa oleifera utilizando o software SAPL, a partir de plântulas obtidas pelo teste de germinação, pelo teste de germinação de sementes submetidas ao teste de envelhecimento acelerado tradicional e pelo teste de germinação de sementes submetidas ao teste de envelhecimento acelerado com solução salina de $\mathrm{NaCl}$, na segunda época de avaliação

Table 4 - Shoot (SL), first root (FRL), total seedling (TSL) length; Uniformity (UI), growth (GI), and vigor (VI) indices from four lots of Moringa oleifera seeds using SAPL software from seedlings obtained by germination test, germination test from seeds submitted to traditional accelerated aging, and from seeds submitted to accelerated aging using $\mathrm{NaCl}$ saline water, at the second evaluation time.

\begin{tabular}{|c|c|c|c|c|c|c|}
\hline \multicolumn{7}{|c|}{ Teste de germinação } \\
\hline \multirow{2}{*}{ Lote } & CPA & CRP & CPT & \multirow{2}{*}{ IU } & \multirow{2}{*}{ IC } & \multirow{2}{*}{ IV } \\
\hline & \multicolumn{3}{|c|}{$\mathrm{Cm}$} & & & \\
\hline 1 & $5,27 \mathrm{~b}$ & $6,92 \mathrm{~b}$ & $12,19 \mathrm{~b}$ & $789,88 \mathrm{~b}$ & $478,10 \mathrm{a}$ & $571,63 \mathrm{a}$ \\
\hline 2 & $4,23 \mathrm{~b}$ & $5,67 \mathrm{~d}$ & $9,90 \mathrm{c}$ & $835,63 \mathrm{a}$ & $389,60 \mathrm{a}$ & $523,41 \mathrm{a}$ \\
\hline 3 & $6,32 \mathrm{a}$ & $8,20 \mathrm{a}$ & $14,53 \mathrm{a}$ & $777,21 \mathrm{bc}$ & 419,85 a & $527,06 \mathrm{a}$ \\
\hline 4 & $5,36 \mathrm{~b}$ & $6,08 \mathrm{c}$ & $11,44 \mathrm{~b}$ & $752,63 \mathrm{c}$ & $411,75 \mathrm{a}$ & $514,01 \mathrm{a}$ \\
\hline $\mathbf{F}$ & $9,16^{*}$ & $275,55^{* *}$ & $72,92^{* *}$ & $25,58^{* * x}$ & $1,00^{\text {ns }}$ & $0,99^{\text {ns }}$ \\
\hline dms & 1,36 & 0,28 & 0,94 & 28,91 & 15,83 & 107,88 \\
\hline $\mathrm{CV} \%$ & 13,34 & 2,00 & 3,75 & 1,75 & 17,75 & 9,62 \\
\hline \multicolumn{7}{|c|}{ Teste de envelhecimento acelerado tradicional } \\
\hline \multirow{2}{*}{ Lote } & CPA & CRP & CPT & \multirow{2}{*}{ IU } & \multirow{2}{*}{ IC } & \multirow{2}{*}{ IV } \\
\hline & & $\mathrm{Cm}$ & & & & \\
\hline 1 & $4,70 \mathrm{~b}$ & $6,05 \mathrm{a}$ & $10,76 \mathrm{ab}$ & $808,82 \mathrm{~b}$ & 403,70 a & $525,20 \mathrm{a}$ \\
\hline 2 & $3,80 \mathrm{~b}$ & $7,04 \mathrm{a}$ & $10,84 \mathrm{ab}$ & $874,56 \mathrm{ab}$ & $431,50 \mathrm{a}$ & $557,56 \mathrm{a}$ \\
\hline 3 & $6,28 \mathrm{a}$ & $6,04 \mathrm{a}$ & $12,32 \mathrm{a}$ & $690,98 \mathrm{c}$ & $459,05 \mathrm{a}$ & $528,63 \mathrm{a}$ \\
\hline 4 & $4,40 \mathrm{~b}$ & $2,90 \mathrm{~b}$ & $7,29 \mathrm{~b}$ & $881,67 \mathrm{a}$ & 340,05 a & $502,54 \mathrm{a}$ \\
\hline $\mathbf{F}$ & $12,15^{* *}$ & $5,44^{*}$ & $4,11^{*}$ & $45,91^{* *}$ & $1,67^{\mathrm{ns}}$ & $0,88^{\mathrm{ns}}$ \\
\hline $\mathrm{dms}$ & 1,36 & 3,03 & 4,38 & 51,88 & 16,52 & 100,6 \\
\hline CV \% & 9,86 & 26,78 & 20,34 & 3,06 & 19,26 & 9,07 \\
\hline
\end{tabular}


Tabela 4 - Conclusão ...

Table 4 - Conclusion ...

\begin{tabular}{|c|c|c|c|c|c|c|}
\hline \multicolumn{7}{|c|}{ Teste de envelhecimento acelerado com solução salina } \\
\hline \multirow{2}{*}{ Lote } & CPA & CRP & $\mathrm{CPT}$ & \multirow{2}{*}{ IU } & \multirow{2}{*}{ IC } & \multirow{2}{*}{ IV } \\
\hline & & $\mathrm{Cm}$ & & & & \\
\hline 1 & $5,31 \mathrm{a}$ & $7,12 \mathrm{a}$ & $12,43 \mathrm{ab}$ & $768,25 b$ & $489,10 \mathrm{ab}$ & $572,84 \mathrm{a}$ \\
\hline 2 & $3,80 \mathrm{~b}$ & $7,04 \mathrm{a}$ & $10,84 \mathrm{~b}$ & 874,56 a & $445,73 \mathrm{~b}$ & $574,37 \mathrm{a}$ \\
\hline 3 & $5,53 \mathrm{a}$ & $7,52 \mathrm{a}$ & 13,06 a & $756,78 \mathrm{~b}$ & $514,50 \mathrm{a}$ & $587,18 \mathrm{a}$ \\
\hline 4 & $5,39 \mathrm{a}$ & $7,84 \mathrm{a}$ & $13,23 \mathrm{a}$ & $780,10 \mathrm{~b}$ & 513,08 a & $593,18 \mathrm{a}$ \\
\hline $\mathbf{F}$ & $10,1^{* *}$ & $3,12^{\text {ns }}$ & $7,08^{* *}$ & $13,86^{* *}$ & $4,38^{*}$ & $1,52^{\mathrm{ns}}$ \\
\hline $\mathrm{dms}$ & 1,06 & 0,88 & 1,71 & 60,83 & 6,44 & 33,65 \\
\hline CV \% & 10,17 & 5,69 & 6,6 & 3,64 & 6,25 & 2,75 \\
\hline
\end{tabular}

Fonte: Autores (2020)

Em que: médias seguidas pela mesma letra não diferem estatisticamente entre si pelo teste de Tukey $(P<0,05) ;{ }^{*}$ significativo em nível de $1 \%$ de probabilidade $(\mathrm{p}<.01)$; ${ }^{*}$ significativo em nível de $5 \%$ de probabilidade $(.01=<\mathrm{p}<.05) ;{ }^{\text {ns }}$ não significativo $(\mathrm{p}>=.05)$.

Os resultados da avaliação das plântulas obtidas após o décimo segundo dia de instalação do teste de germinação pelo método tradicional e analisadas pelo SAPL estão apresentados na Tabela 5. O CPA apresentou superioridade do lote $1 \mathrm{em}$ relação aos demais lotes. O ranqueamento dos lotes foi semelhante para o CRP e o CPT, nos quais os lotes de maiores comprimentos foram os lotes 1 e 3. Para o IU, o lote 2 foi o que apresentou maior uniformidade de desenvolvimento, enquanto para o lote 3, esse valor foi menor. Quando analisados o IC e o IV, observou-se que o lote 3 apresentou resultados superiores sobre os demais lotes. Mediante as análises de plântulas obtidas pelo teste de germinação, após o décimo segundo dia, verificou-se superioridade do lote 3 , quando comparado aos demais.

No teste com sementes de moringa submetidas ao envelhecimento acelerado tradicional, analisadas pelo SAPL, após doze dias da instalação do teste (Tabela 5), observa-se uma exatidão no ranqueamento na avaliação do CPA, CRP, IU, IC e IV. O lote 1 foi superior na maioria das avaliações (CPA, CRP, CPT, IC e IV). Constatou-se relação entre o ranqueamento dos lotes e o índice de crescimento (IC), o índice de vigor (IV), a porcentagem de germinação e o IVG. O lote 2 foi o que possuiu maior índice de uniformidade (IU). O envelhecimento acelerado tradicional, na terceira época de avaliação, novamente foi o teste que apresentou maior sensibilidade no ranqueamento dos lotes.

Estudos realizados com sementes Tabernaemontana hystrix (leiteira) (MORAES et al., 2016) e Poincianella pyramidalis (catingueira) (LIMA et al., 2012) destacaram a eficiênciado envelhecimento acelerado tradicional e a baixa sensibilidade do envelhecimento acelerado com solução salina de $\mathrm{NaCl}$, para avaliar o vigor de sementes. Entetanto, estudos com sementes de espécies agrárias de Capsicum annuum L. (pimentão) (PANOBIANCO; MARCOS FILHO, 1998) e de Brassica oleracea L. (couve-brócolos) (MARTINS et al., 2002) observaram que testes usando o envelhecimento acelerado $\left(41^{\circ} \mathrm{C}\right.$ por $\left.72 \mathrm{~h}\right)$ com solução salina foram mais eficazes na detecção de diferenças de qualidade de lotes que o envelhecimento acelerado tradicional. Contudo, em sementes de BixaorellanaL. (urucum), o envelhecimento acelerado com água ou solução salina por 72 horas ou 96 horas foram eficientes para ranquear o vigor(FERREIRA; NOVEMBRE, 2016).

Os resultados dos estudos com sementes de moringa submetidas ao envelhecimento acelerado com solução salina de $\mathrm{NaCl}$, analisadas pelo SAPL, com doze dias (Tabela 5), acusaram 
inferioridade do CPA do lote 4, quando comparado com os demais lotes e destacou os lotes $1 \mathrm{e}$ 3 como os melhores. Entretanto, quando analisado o CRP, percebe-se discriminação de vigor, sugerindo os lotes com comportamento superior (lote 4), intermediários (lote 1 e 2) e inferior (lote 3). Entretanto, quando se avaliou o IC, o lote 4 apresentou resultados inferiores aos dos demais lotes. O IV apresentou pouca sensibilidade para diferenciação do vigor, pois só conseguiu ranquear os melhores lotes (lote 1 e 3 ) e os piores lotes (lote 2 e 4).

Tabela 5 - Comprimento da parte aérea (CPA), da raiz primária (CRP) da plântula total (CPT), índices de uniformidade (IU), crescimento (IC) e vigor (IV) de quatro lotes de sementes de Moringa oleifera utilizando o software SAPL, a partir de plântulas obtidas pelo teste de germinação, pelo teste de germinação de sementes submetidas ao teste de envelhecimento acelerado tradicional e pelo teste de germinação de sementes submetidas ao teste de envelhecimento acelerado com solução salina de $\mathrm{NaCl}$, na terceira época de avaliação

Table 5 - Shoot (SL), first root (FRL), total seedling (TSL) length; Uniformity (UI), growth (GI), and vigor (VI) indices from four lots of Moringa oleifera seeds using SAPL software from seedlings obtained by germination test, germination test from seeds submitted to traditional accelerated aging, and from seeds submitted to accelerated aging using $\mathrm{NaCl}$ saline water at the third evaluation time

\begin{tabular}{|c|c|c|c|c|c|c|}
\hline \multicolumn{7}{|c|}{ Teste de germinação } \\
\hline \multirow{2}{*}{ Lote } & CPA & CRP & CPT & \multirow{2}{*}{ IU } & \multirow{2}{*}{ IC } & \multirow{2}{*}{ IV } \\
\hline & \multicolumn{3}{|c|}{$\mathrm{Cm}$} & & & \\
\hline 1 & $7,51 \mathrm{a}$ & $7,11 \mathrm{a}$ & $14,63 \mathrm{a}$ & $639,12 \mathrm{bc}$ & $414,80 \mathrm{c}$ & $482,10 \mathrm{c}$ \\
\hline 2 & $5,16 \mathrm{~b}$ & $6,05 \mathrm{~b}$ & $11,21 \mathrm{~b}$ & $764,07 \mathrm{a}$ & $404,67 \mathrm{c}$ & $512,49 \mathrm{~b}$ \\
\hline 3 & $6,10 \mathrm{ab}$ & $7,50 \mathrm{a}$ & $13,60 \mathrm{a}$ & $602,65 c$ & $632,13 \mathrm{a}$ & $623,29 \mathrm{a}$ \\
\hline 4 & $6,10 \mathrm{ab}$ & $6,04 \mathrm{~b}$ & $12,15 \mathrm{~b}$ & $676,55 \mathrm{~b}$ & $467,80 \mathrm{~b}$ & $530,42 \mathrm{~b}$ \\
\hline $\mathbf{F}$ & $8,25^{* \star}$ & $11,7^{\text {** }}$ & $20,34^{\text {** }}$ & $30,23^{* *}$ & $294,64^{\text {** }}$ & $740,09^{* *}$ \\
\hline $\mathrm{dms}$ & 1,41 & 0,91 & 1,41 & 52,87 & 2,57 & 25,68 \\
\hline CV \% & 10,86 & 6,53 & 5,21 & 3,75 & 2,55 & 3,54 \\
\hline \multicolumn{7}{|c|}{ Teste de envelhecimento acelerado tradicional } \\
\hline \multirow{2}{*}{ Lote } & CPA & CRP & CPT & IU & IC & IV \\
\hline & \multicolumn{3}{|c|}{$\mathrm{Cm}$} & & & \\
\hline 1 & 6,47 a & $9,01 \mathrm{a}$ & $15,48 \mathrm{a}$ & $720,95 \mathrm{c}$ & $612,5 \mathrm{a}$ & $645,01 \mathrm{a}$ \\
\hline 2 & $3,59 \mathrm{~d}$ & $7,58 \mathrm{~b}$ & $11,18 \mathrm{~b}$ & 886,39 a & $327,2 \mathrm{c}$ & $494,96 \mathrm{c}$ \\
\hline 3 & $5,91 \mathrm{~b}$ & $5,13 \mathrm{c}$ & $11,04 \mathrm{~b}$ & $646,21 d$ & $450,1 \mathrm{~b}$ & $508,91 \mathrm{~b}$ \\
\hline 4 & $4,97 \mathrm{c}$ & $2,94 \mathrm{~d}$ & $7,92 \mathrm{c}$ & $738,19 \mathrm{~b}$ & $268,4 \mathrm{~d}$ & $409,34 \mathrm{~d}$ \\
\hline $\mathbf{F}$ & $356,98^{* k}$ & $1434,44^{* *}$ & $932,24^{* *}$ & $1420,21^{* *}$ & $1188,79^{* k}$ & $1645,41^{* *}$ \\
\hline dms & 0,27 & 0,29 & 0,42 & 11,2 & 1,86 & 12,85 \\
\hline CV \% & 2,54 & 2,3 & 1,78 & 0,71 & 2,17 & 0,83 \\
\hline
\end{tabular}


Tabela 5 - Conclusão ...

Table 5 - Conclusion ...

\begin{tabular}{|c|c|c|c|c|c|c|}
\hline \multicolumn{7}{|c|}{ Teste de envelhecimento acelerado com solução salina } \\
\hline \multirow{2}{*}{ Lote } & CPA & CRP & CPT & \multirow[t]{2}{*}{ IU } & \multirow[t]{2}{*}{ IC } & \multirow[t]{2}{*}{ IV } \\
\hline & & $\mathrm{Cm}$ & & & & \\
\hline 1 & $5,98 \mathrm{a}$ & $6,97 \mathrm{~b}$ & $12,96 \mathrm{a}$ & $721,69 a b$ & $559,03 \mathrm{a}$ & $607,83 \mathrm{a}$ \\
\hline 2 & $5,21 \mathrm{ab}$ & $8,35 \mathrm{a}$ & $13,54 \mathrm{a}$ & $790,09 \mathrm{ab}$ & 547,98 a & $620,61 \mathrm{a}$ \\
\hline 3 & $5,97 \mathrm{a}$ & $6,78 \mathrm{~b}$ & $12,75 \mathrm{a}$ & $708,09 \mathrm{~b}$ & $502,23 \mathrm{ab}$ & $563,99 \mathrm{~b}$ \\
\hline 4 & $4,59 \mathrm{~b}$ & $6,48 \mathrm{~b}$ & $11,72 \mathrm{a}$ & $793,53 \mathrm{a}$ & $474,97 \mathrm{~b}$ & $569,84 \mathrm{~b}$ \\
\hline $\mathbf{F}$ & $4,49^{*}$ & $7,25^{\star *}$ & $1,82^{\mathrm{ns}}$ & $4,87^{*}$ & $6,65^{* *}$ & $23,87^{\text {t* }}$ \\
\hline $\mathrm{dms}$ & 1,32 & 1,29 & 2,36 & 85,28 & 6,46 & 23,99 \\
\hline CV \% & 11,64 & 8,62 & 8,82 & 5,38 & 5,91 & 1,93 \\
\hline
\end{tabular}

Fonte: Autores (2020)

Em que: médias seguidas pela mesma letra não diferem estatisticamente entre si pelo teste de Tukey $(P<0,05)$; ${ }^{* *}$ significativo em nível de $1 \%$ de probabilidade $(\mathrm{p}<.01) ;{ }^{*}$ significativo em nível de $5 \%$ de probabilidade $(.01=<\mathrm{p}<$ $.05) ;{ }^{\text {ns }}$ não significativo $(\mathrm{p}>=.05)$.

Os resultados obtidos para o comprimento das plântulas de moringa, após 10 e 12 dias da instalação do teste de germinação pelo método tradicional (Tabelas 3 e 4), corroboram com Dan et al. (1987), os quais afirmaram que as sementes vigorosas originam plântulas com maior taxa de crescimento, em função da maior capacidade de transformação do suprimento de reservas dos tecidos de armazenamento e da maior incorporação destes pelo eixo embrionário. Dessa forma, as amostras com maiores valores de comprimento médio de plântulas normais ou das partes destas são consideradas mais vigorosas, como é o caso dos lotes 1 e 3.

Os maiores índices de velocidade de germinação obtidos neste estudo estão relacionados com os maiores comprimentos de plântulas (Tabela 1).Essa relação amplia a possibilidade de sucesso durante o estabelecimento da plântula, visto que o crescimento rápido - tanto da raiz primária quanto da parte aérea - viabiliza o aproveitamento mais rápido das reservas hídricas e nutricionais do solo, bem como o melhor desenvolvimento dos processos fisiológicos. As plântulas com maiores índices de velocidade de emergência iniciam o processo fotossintético mais rapidamente, mantendo os maiores comprimentos também ao longo do período de desenvolvimento, devido ao maior tamanho inicial que as de menor vigor, o que de fato é fundamental para produção de mudas (SCHUCH et al., 1999), que corrobora os resultados obtidos no presente trabalho. O comprimento das plântulas, similarmente, foi o método mais sensível para diferenciar lotes de sementes de Amburana cearensis (cumaru) (GUEDES et al., 2015), bem como o uso dos testes de comprimento de plântulas e de emergência em campo foram indicados por Guedes et al. (2009b) como viáveis para separação de lotes de sementes da espécie florestal Erythrina velutina Willd (mulungu).

Há uma tendência consistente, a partir dos resultados obtidos neste trabalho, para a maioria dos testes de classificação dos níveis de vigor de sementes, incluindo com o uso do SAPL, e, de modo destacado, os testes de germinação, índice de velocidade de germinação, emergência, de que o lote 1 foi o classificado como o mais vigoroso.

Com o uso do software SAPL, o envelhecimento acelerado tradicional foi o teste que apresentou melhores resultados para o ranqueamento de lotes de sementes de moringa, de acordo com sua qualidade fisiológica, especialmente para o comprimento da parte aérea, da raiz primária, da plântula total e o índice de uniformidade. 


\section{Conclusão}

As sementes dos lotes 1 e 3 apresentam a melhor qualidade fisiológica. O software SAPL é eficiente para avaliar a qualidade de sementes de moringa, a partir de imagens de plântulas obtidas no teste de germinação ou no teste de envelhecimento acelerado tradicional.

\section{Referências}

BAALBAKI, R.et al. Seed vigor testing handbook. Ithaca: Association of Official Seed Analysts, 2009. $341 \mathrm{p}$.

BENNETT, M. A. Saturated salt accelerated aging (SSAA) and other vigor tests for vegetable seeds. In: INTERNATIONAL SEED SEMINAR, 2002, Santiago. Proceedings [...]. Santiago: PUC de Chile, 2002. p. 188-193.

BEZERRA, A. M. E. et al. Germinação de sementes e desenvolvimento de plântulas de moringa (Moringa oleifera Lam.) em função do peso da semente e do tipo de substrato. Horticultura Brasileira, Brasília, v. 22, n. 2, p. 295-299, 2004.

BRASIL. Ministério da Agricultura, Pecuária e Abastecimento. Regras para análise de sementes. Brasília: Mapa; ACS, 2009. 398 p.

CARVAlHO, N. M.; NAKAGAWA, J. (ed.) Sementes: ciências, tecnologia e produção. 5. ed. Jaboticabal: FUNEP, 2012. 588 p.

DAN, E. L. et al. Transferência de matéria seca como modo de avaliação do vigor de sementes de soja. Revista Brasileira de Sementes, Brasília, v. 9, n. 3, p. 45-55, 1987.

DORNELAS, M. C.; LOBO, C. A.; VIEIRA, I. G. Evaluation of Eucalyptus spp. seedling size following germination using computer-aided analysis of digital images. Scientia Forestalis, Picaracicaba, v. 2,n. 68, p. 125-130, 2005.

FANTI, S. C.; PEREZ, S. C. J. G. A. Efeitos do envelhecimento precoce no vigor de sementes de Chorisia speciosa St. Hil. - Bombacaceae. Revista Árvore, Viçosa, MG, v. 3, n. 1, p. 345-352, 2005.

FERREIRA, R. L.; NOVEMBRE, A. D. L. C. Estimativa do vigor das sementes e das plântulas de Bixaorellana L. Revista Ciência Agronômica, Fortaleza, v. 47, n. 1, p. 101-107, 2016.

GOMES JUNIOR, F. G. et al. Evaluation of priming effects on sweet corn seeds by SVIS. Seed Technology, Lincoln, v. 31, n. 1, p. 95-100, 2009.

GUEDES, R. S. et al. Avaliação do potencial fisiológico de sementes de Amburana cearensis (Allemão) A.C. Smith. Bioscience Jornal, Uberlândia, v. 29, n. 4, p. 859-866, 2013.

GUEDES, R. S. et al. Resposta fisiológica de sementes de Erythrina velutina Willd. ao envelhecimento acelerado. Semina: Ciências Agrárias, Londrina, v. 30, n. 2, p. 323-330, 2009a.

GUEDES, R. S. et al. Testes de vigor na avaliação da qualidade fisiológica de sementes Erythrina velutina Willd. (Fabaceae - Papilionoideae). Ciência e Agrotecnologia, Lavras, v. 33, n. 5, p. 1360$1365,2009 b$.

GUEDES, R. S. et al. Teste de comprimento de plântula na avaliação da qualidade fisiológica de sementes de Amburana cearensis (Allemão) A.C. Smith. Semina: Ciências Agrárias, Londrina, v. 36, n. 4, p. 2373-2382, 2015.

HOFFMASTER, A. L. et al. An automated system for vigour testing three-day-old soybean seedling. Seed Science and Technology, Zurich, v. 33, n. 1, p. 701-713, 2003.

JIANHUA, Z.; MCDONALD, M. B. The saturated salt accelerated aging test for small-seeded 
crops.Seed Science and Technology, Zurich, v. 25, n. 1, p. 123-131, 1996.

KOBORI, N. N. et al. Effects of gamma radiation on physiological and phytosanitary qualities of Brazilian Castor Bean seeds, Ricinus communis (cv. IAC Guarani). Research Journal of Seed Science, New York, v. 3, n. 2, p. 70-81, 2010.

LIMA, C. R. et al. Physiological maturity of fruits and seeds of Poincianella pyramidalis (Tul.) L.P. Queiroz. Revista Brasileira de Sementes, Londrina, v. 34, n. 2, p. 231-240, 2012.

MAGUIRE, J. D. Speed of germination aid in selection and evaluation for seedling emergence and vigor. Crop Science, Madison, v. 2, n. 2, p. 176-177, 1962.

MARCOS FILHO, J. Fisiologia de sementes de plantas cultivadas. Londrina: ABRATES, 2015a. $659 \mathrm{p}$.

MARCOS FILHO, J. Seed vigor testing: an overview of the past, present and future perspective. Scientia Agricola, Piracicaba, v. 72, n. 4, p. 363-374, 2015b.

MARCOS FILHO, J. et al. Métodos para avaliação do vigor de sementes de soja, incluindo a análise computadorizada de imagens. Revista Brasileira de Sementes, Brasília, v. 31, n. 1, p. 102-112, 2009.

MARTINS, C. et al. Comparação entre métodos para a avaliação do vigor de lotes de sementes de couve-brócolos (Brassica olerace L. var. itálica Plenk). Revista Brasileira de sementes, Brasília, v. 24, n. 2, p. 96-101, 2002.

MEDEIROS, M. L. S. Testes para a avaliação da qualidade fisiológica de sementes de Moringa oleifera Lam. 2015. Dissertação (Mestrado em Ciências Florestais) - Universidade Federal do Rio Grande do Norte, Macaíba, 2015.

MORAES, C. E. et al. qualidade fisiológica de sementes de Tabernaemontana fuchsiaefolia A. DC em função do teste de envelhecimento acelerado. Ciência Florestal, Santa Maria, v. 26, n. 1, p. 213-223, 2016.

NAKAGAWA, J. Teste de vigor baseados na avaliação das plântulas. In: VIEIRA, R. D.; CARVALHO, N. M. Teste de vigor em sementes. Jaboticabal: FUNEP, 1999. p. 49-85.a

NAKAGAWA, J. Testes de vigor baseados no desempenho das plântulas. In: KRZYZANOSKI, F. C.; VIEIRA, R. D.; FRANÇA NETO, J. B. (ed.). Vigor de sementes: conceitos e testes. Londrina: ABRATES, 1999. p. 2.1-2. 24. b

OAKLEY, K. et al. Computeraided digital image analysis of seedling size and growth rate for assessing seed vigour in Impatiens. Seed Science and Technology, Zurich, v. 32, n. 3, p. 907-915, 2003.

PACHECO; M. V. et al. Physiological quality evaluation of the radii Schinus terebinthifolius seeds. Revista Brasileira de Sementes, Londrina, v. 33, n. 4, p. 762-767, 2011.

PANOBIANCO, M.; MARCOS FILHO, J. Comparação entre métodos para avaliação da qualidade fisiológica de sementes de pimentão. Revista Brasileira de Sementes, Brasília, v.20, n.2, p.306310, 1998.

PONTES, C. A. et al. Influência da temperatura de armazenamento na qualidade das sementes de Caesalpinia peltophoroides Benth. (sibipiruna). Revista Árvore, Viçosa, MG, v. 30, n. 1, p. 43-48, 2006.

ROCHA, T. G. F. Qualidade fisiológica de sementes e plântulas de Moringa oleifera LAM. por meio da técnica de análise de imagens. 2015. Dissertação (Mestrado em Ciências Florestais) Universidade Federal do Rio Grande do Norte, Macaíba, 2015.

ROCHA, T. G. F. et al. Vigor de sementes de moringa pelo teste de emissão da raiz primária. 
Tecnologia \& Ciência Agropecuária, João Pessoa, v. 10, n. 5, p. 63-68, 2016.

SAKO, J. F. et al. A system for automated seed vigor assessment. Seed Science and Technology, Zurich, v. 29, n. 3, p. 625-636, 2001.

SAS Institute. Statistical Analysis System: getting stared with the SAS learning. Cary, 2000.81 p.

SILVA, V. N.; CICERO, S. M. Image seedling analysis to evaluate tomato seed physiological potential. Revista Ciência Agronômica, Fortaleza, v. 45, n. 2, p. 327-334, 2014.

SCHUCH, L. O. B. et al. Crescimento em laboratório de plântulas de aveia preta (Avena strigosa Schreb) em função do vigor das sementes. Revista Brasileira de Sementes, Brasília, v. 21, n. 1, p. 229-234, 1999.

VIEIRA, R. D.; CARVALHO, N. M. Testes de vigor em sementes. Jaboticabal: FUNEP, 1994. 164 p. 